\title{
Case Report \\ Pneumonia Presenting as Abdominal Pain in a Pediatric Patient
}

\author{
Sarah MacPhee', Erin Killorn MD, FRCPC ${ }^{2}$ \\ ${ }^{1}$ Class of 2016, Faculty of Medicine, Dalhousie University \\ ${ }^{2}$ Department of Pediatric Emergency Medicine, Dalhousie University
}

\begin{abstract}
Although clinically pneumonia is generally manifested by fever, tachypnea, dyspnea, and increased work of breathing, the pediatric presentation can vary significantly. Pneumonia may present as acute abdominal pain in children, which can go unrecognized on a patient's initial evaluation and even be mistaken for a surgical condition. Streptococcus pneumoniae is the most common cause of bacterial pneumonia in the pediatric population and is frequently preceded by an upper respiratory infection. Described is the case of a six-year-old female with severe pneumococcal infection and pleural effusion following a history of upper respiratory illness. The patient was initially transferred for assessment as a potential case of appendicitis.
\end{abstract}

\section{Case presentation}

A six-year-old female presented to the IWK Emergency Department (ED), with a chief complaint of abdominal pain that was persistent at rest and worse with inspiration. Several hours earlier the patient had presented to a community ED outside the city limits and was transferred to the IWK to rule out appendicitis. This was suspected due to new onset abdominal pain with right lower quadrant (RLQ) tenderness. In children and adolescents, abdominal pain that localizes to the RLQ and is worse with movement is suspicious for appendicitis.

On history, the patient had a 10-day history of fever, cough and sore throat. For the five days preceding her presentation to the ED, she had been noted to have difficulty breathing, anorexia and lethargy with single episodes of both vomiting and diarrhea three days before her presentation. She also had bilateral ear drainage with decreased hearing. She had presented to the IWK ED a week prior to her latest admission, with a chief complaint of fever with associated nasal congestion, vomiting, and diarrhea. Her posterior oropharynx was noted to be red on examination. A urinalysis, urine culture, and throat swab for Group A Streptococcus was completed and was unremarkable. She was discharged with the presumptive diagnosis of a viral infection, with instructions to return if the fever persisted.

Past medical history revealed that she had not been vaccinated past six months of age, as she had a negative reaction to her six-month immunizations while living in Ontario. The specifics of this adverse reaction are not known.
On examination, the patient had a temperature of $39^{\circ} \mathrm{C}$, a respiration rate of 48 , pulse of 150 , blood pressure of $105 / 62 \mathrm{~mm} \mathrm{Hg}$, and an oxygen saturation of $93 \%$ on room air. Her abdomen was soft but diffusely tender to palpation. Inspection revealed copious bilateral purulent discharge from her ears. Decreased air entry on the right side with fine inspiratory crackles was heard on auscultation. The remainder of her examination was unremarkable. The initial impression was that her abdominal pain could be attributable to pneumonia rather than appendicitis as initially presumed. A chest $\mathrm{X}$-ray and blood work were ordered, the patient was placed on oxygen for comfort, and a bolus of normal saline was initiated intravenously (IV).

The initial chest X-ray revealed a significant diffuse opacity involving the entire right lobe as well as a rightsided effusion. Initial laboratory findings showed a WBC count of $38.4 \times 10^{9} / \mathrm{L}$, and a neutrophil count of $33.84 \times 10^{9} / \mathrm{L}$ with $26 \%$ band neutrophils. Her venous $\mathrm{pH}$ was only slightly elevated at 7.48 with a $\mathrm{PCO}_{2}$ of 33.5 and $\mathrm{HCO}_{3}$ of 24.7. Electrolytes were within normal limits. Swabs for Mycoplasma and Chlamydia pneumonia were negative, as were blood cultures and nasopharyngeal aspirates for Respiratory syncytial virus and Influenza.

The patient was admitted to hospital, and started on IV cefotaxime, oral azithromycin, as well as $\mathrm{Ciprodex}^{\circ}$ (ciprofloxacin and dexamethasone) ear-drops for what was determined to be bilaterally perforated eardrums. A chest ultrasound done upon admission confirmed the presence of a pleural effusion. A chest tube was inserted two days later that drained in excess of $2 \mathrm{~L}$ of total fluid over four days. Pleural samples revealed bloody fluid with a high WBC count of $3122 / \mathrm{mm}^{3}$, 
composed of $96 \%$ neutrophils and $4 \%$ bands. Pleural fluid later tested positive for Streptococcus pneumonia by polymerase chain reaction.

Prior to chest tube placement, the patient remained febrile and tachypneic requiring supplemental oxygen and morphine for pain management. Once the fluid was removed, the patient began to improve significantly. A follow-up chest X-ray was performed to confirm placement of the chest tube and showed partial resolution of the pneumonia and associated effusion. At this time the decision to remove the chest tube was made. A chest X-ray also showed residual heterogeneous changes in the right upper lobe, which were thought to possibly be a pneumatocele or necrotic lung changes in the parenchyma.

The patient remained afebrile 48 hours after removal of the chest tube and was transitioned to oral antibiotics. The morphine infusion and supplemental oxygen were discontinued, and the patient was discharged home with a prescription to complete a further seven-day course of oral cefprozil. Follow-up planning included a repeat chest X-ray and reassessment by the family doctor. An outpatient consultation was arranged with pediatrics to discuss resolution of the pneumonia as well as noted chronic health issues including obesity (weight exceeded the 97th percentile for six years of age), anemia (initial blood work in ED revealed a hemoglobin of $106 \mathrm{~g} / \mathrm{L}$ with a $\mathrm{MCV}$ of 74.3), and vaccination status.

\section{Discussion}

Pneumonia is the single most common disease causing death in children worldwide under the age of five. ${ }^{1}$ Streptococcus pneumoniae is the most common bacterial cause of pneumonia in the pediatric population. ${ }^{2}$ It is frequently preceded by minor upper respiratory infection, which can be characterized by low-grade fever and rhinorrhea. ${ }^{3}$

Although clinically pneumonia is generally manifested by fever, tachypnea, dyspnea, and increased work of breathing, the pediatric presentation can vary significantly, often with more subtle signs and symptoms in infants and young children. ${ }^{4}$ The initial presentation of pneumonia in children aged five to 10 years can be fever and leukocytosis, without cough or respiratory distress. ${ }^{5}$

Upper lobe pneumonia may present as nuchal rigidity. ${ }^{5}$ Basal pneumonia may present with significant abdominal pain; the diaphragm is innervated by intercostal nerves that also supply the muscles, skin and peritoneum of the abdominal wall, and thus diaphragmatic irritation due to basal pneumonia may present as an acute abdominal pain. ${ }^{6,7}$ An alternative explanation for the abdominal pain is that pneumonia often coexists with mesenteric lymphadenitis. ${ }^{7}$ The inflammation of mesenteric lymph nodes mimics appendicitis since the inflamed nodes are typically clustered in the right lower quadrant. ${ }^{8}$

This variation in presentation poses a diagnostic challenge for clinicians, especially those who may not deal routinely with pediatric patients. The differential diagnosis for abdominal pain in children ages six to 11 years includes gastroenteritis, appendicitis, constipation, urinary tract infection, pneumonia, mesenteric lymphadenitis among other things. ${ }^{8}$ Pneumonia accounts for $1.89 \%$ of all children who present initially with abdominal pain in an emergency setting. ${ }^{9}$ In light of this low prevalence, it has been recommended that children under the age of 12 who present to the ED with acute abdominal pain and in whom an abdominal radiograph is requested, need only undergo a chest radiograph in the presence of cough, fever or other symptoms of an upper respiratory tract infection (URTI). ${ }^{9}$ Additional preliminary tests in the evaluation of children with suspected pneumonia may include a $\mathrm{CBC}$ with differential, serum electrolytes, and acute phase reactants.

S. pneumoniae in particular will manifest itself with fever, non-productive cough, tachypnea, and decreased breath sounds over the affected area. WBC count may be dramatically elevated. If possible, a microbiological diagnosis is recommended in children with severe disease, those with potential complications, and patients requiring hospitalization. Severe disease may be clinically manifested by a temperature of $\geq 38.5^{\circ} \mathrm{C}$, moderate to severe respiratory distress, cyanosis, altered mental status, hypoxemia, tachycardia and poor peripheral perfusion. ${ }^{2}$ Specific diagnosis of pneumococcal pneumonia (including S. pneumonia) in children is usually made through blood or pleural fluid cultures. ${ }^{2}$ However, it is estimated that as few as one-third of the cases of pneumonia can be attributed to a specific etiology. ${ }^{3}$

Treatment with cefotaxime and azithromycin is the suggested empirical antibiotic regimen for severe pneumonia. Use of both a beta-lactam and macrolide antibiotic is recommended due to severe disease requiring a broader spectrum of coverage, especially 
if etiologies other than $S$. pneumonia are being considered. ${ }^{10}$

The most common complication of pneumococcal pneumonia in children is pleural effusion. ${ }^{2}$ Other possible complications include empyema (i.e., infection of the pleural space), pericarditis, endobronchial obstruction, atelectasis and lung abscess formation. ${ }^{11}$ Complications have been correlated with a weight less than the 10th percentile for age, vaccination status, comorbid conditions, anemia, immunodeficiency, as well as the severity of the pneumonia. ${ }^{12}$ Pneumococcal conjugate vaccination has been found effective in reducing the burden of invasive pneumococcal disease, especially for children. ${ }^{13}$ Unvaccinated children are at risk for the more serious strains of pneumococcus that are contained within the conjugated vaccine. ${ }^{14}$

This case represents an important clinical teaching point. The patient's history and exam features should prompt health care workers to consider pneumonia with a high index of suspicion, rather than a surgical condition. Not uncommonly, pediatric pneumonia may present as abdominal pain. ${ }^{6}$ Findings include intermittent fever, decreased breath sounds in the context of a recent upper respiratory illness, and a soft abdomen on examination. This patient's bilateral ear discharge provides a clue as to the primary site of pneumococcal infection. This patient also has risk factors in her presentation for severe pneumococcal disease, including her vaccination status and anemia.

\section{Conclusion}

This case illustrates the importance of being aware of the variety of ways in which pneumonia may present in children. Always consider pneumonia in the differential diagnosis of children with fever and abdominal pain. Those with suggestive findings of pneumonia namely cough, fever, or symptoms of a URTI should be investigated with a chest X-ray to establish whether a pneumonia is present before pursuing surgical causes for abdominal findings.

\section{References}

1. Partnership for Maternal, Newborn \& Child Health. "Child Mortality." Millenium Development Goal (MDG) 4. Revised September 2011. <http://www.who.int/pmnch/media/press_materials/fs/fs_mdg4_ childmortality/en/> (16 December 2014).

2. <http://www.uptodate.com/contents/pneumonia-in-childreninpatient-treatment?source $=$ search_result\&search $=$ pneumonia + childre n\&selectedTitle $=2 \sim 150>$ (20 January 2014).

3. McCracken GH. Diagnosis and management of pneumonia in children. Pediatr Infect Dis J 2000;19:924-8.

4. Bickley LS, Szilagyl PG. Bates' Guide to Physical Exam and History
Taking. 11th ed. Philadelphia: Wolters Kluwer Health with Lippincott Williams \& Wilkins, 2013:842.

5. Barson WJ." Community Acquired Pneumonia in Children: Clinical Features and Diagnosis." 2014. < http://www.uptodate.com/contents/ community-acquired-pneumonia-in-children-clinical-features-anddiagnosis? source $=$ search

6. Drake RL, Vogl AW, Mitchell AWM. Gray's Anatomy for Students. 2nd ed. Philadelphia: Churchhill Livingstone Elsevier; 2010:134-145.

7. Paul SP, Fitz John L. Abdominal pain in children with pneumonia. Nurs Times 2012;108(14-15):21.

8. Leung AKC, Sigalet DL. Acute abdominal pain in children. Am Fam Physician 2003;67:2312-26.

9. Homier V. Prevalence of pneumonia in children under 12 years of age who undergo abdominal radiography in the emergency department. Can J Emerg Med Care 2007;9(5):347-51.

10. Saux NL, Robinson JL. "Pneumonia in healthy Canadian children and youth: Practice Points for management." September 2011. < http://www. cps.ca/documents/position/pneumonia-management-children-youth> (16 December 2014).

11. Centers for Disease Control and Prevention. "Vaccines and Immunizations: Pneumococcal Disease" 2012. <http://www.cdc.gov/ vaccines/pubs/pinkbook/pneumo.html\#schedule> (22 January 2014).

12. Chang $A B$, Mong HO, Perera D, Grimwood K. Improving the diagnosis, management, and outcomes of children with pneumonia: where are the gaps? Frontiers in Pediatrics 2013;1(29):1-6.

13. Tuomanen EI. "Pneumococcal (Streptococcus pneumonia) Conjugate Vaccines in Children." 2014. < http://www.uptodate.com/contents/ pneumococcal-streptococcus-pneumoniae-conjugate-vaccines-inchildren?source $=$ search_result\&search $=$ pneumococcal+vaccine\&select edTitle $=3 \sim 150>$ (21 January 2014).

14. The Children's Hospital of Philidelphia. "Vaccine Education Centre, Pneumococcus Vaccine." 2013. <http://www.chop.edu/service/vaccineeducation-center/a-look-at-each-vaccine/pneumococcus-vaccine. html $>$ (22 January 2014).

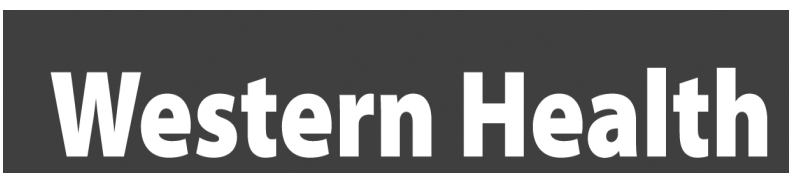

Newfoundland and Labrador

\section{A beautiful place to live and work!}

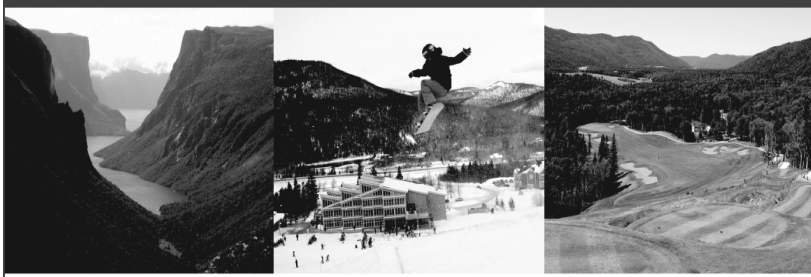

Employment Opportunities!

- Specialists and Family Physicians

- Fee For Service and Salaried Vacancies

Contact Sarah at medicalservices@westernhealth.nl.ca or visit www.westernhealth.nl.ca/physicians for details.

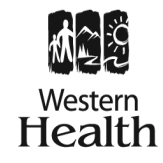

Start living in the Western Health region! 